MSUCL-884

WSU-NP-93-1

\title{
NUCLEAR FLOW EXCITATION FUNCTION
}

\author{
Dietrich Klakow, ${ }^{a, *}$ Gerd Welke, ${ }^{b}$ and Wolfgang Bauer ${ }^{a}$ \\ ${ }^{a}$ National Superconducting Cyclotron Laboratory \\ and Department of Physics and Astronomy, \\ Michigan State University, East Lansing, MI 48824. \\ ${ }^{b}$ Department of Physics and Astronomy, \\ Wayne State University, Detroit, MI 48202.
}

\begin{abstract}
We consider the dependence of collective flow on the nuclear surface thickness in a Boltzmann-Uehling-Uhlenbeck transport model of heavy ion collisions. Well defined surfaces are introduced by giving test particles a Gaussian density profile of constant width. Zeros of the flow excitation function are as much influenced by the surface thickness as the nuclear equation of state, and the dependence of this effect is understood in terms of a simple potential scattering model. Realistic calculations must also take into account medium effects for the nucleon-nucleon cross section, and impact parameter averaging. We find that balance energy scales with the mass number as $A^{-y}$, where $y$ has a numerical value between 0.35 and 0.5 , depending on the assumptions about the in-medium nucleon-nucleon cross section.
\end{abstract}

PACS Indices: 25.70.-z, 02.70.Lq, 21.65.+f 
Broadly speaking, nuclear collective flow in a heavy ion collision is the deflection of nuclear matter perpendicular to the beam axis during the course of the reaction. Experimentally, one observes that flow disappears at a well defined beam energy [1][5], the so called balance energy $E_{B a l}$, whose value depends on the system and impact parameter range being considered. These zeros in the flow excitation function were predicted by the Boltzmann-Uehling-Uhlenbeck (BUU) transport model [6, [], and an analysis of scale invariant quantities [8], and may be understood as an overall cancellation of the attractive part of the mean field interaction with repulsive contributions from the mean field and collisional kinetic pressure. Thus, as has been shown explicitly for BUU simulations in Ref. [3], $E_{B a l}$ is expected to depend on both the nuclear equation of state and the magnitude of the in-medium nucleon-nucleon cross section. By making a systematic study of the balance energy as a function of the nuclear mass, one therefore hopes to gain insight into these properties. However, other parameters might well influence the balance energy. In particular, we wish to investigate in this note the effect of finite nuclear surface thicknesses and impact parameter variations on $E_{B a l}$. We begin by defining the flow variable to be used here, and point out the importance of obtaining well-defined nuclear surfaces that are independent of the grid size used to compute density gradients. We then show how the strong surface dependence of flow in a Vlasov simulation may be understood in terms of a simple potential scattering model. This dependence persists for full BUU calculations that include a non-zero collision integral. Lastly, we consider the effect on the balance energy when the mass number and impact parameter are varied.

To analyze flow quantitatively in experiments one of the main problems that has to be addressed is the determination of the reaction plane (see, for example, Refs. [9]-11]). In a model calculations, on the other hand, knowledge of the reaction plane immediately allows one to define a flow variable such as the average in-plane transverse momentum

$$
\left\langle w P_{x}\right\rangle=\frac{1}{N} \sum_{i=1}^{N} w_{i} P_{i}^{x}
$$


where the weight $w_{i}=1$ or -1 if the test particle $i$ is emitted into the forward or backward center-of-mass hemispheres, respectively, and $P_{i}^{x}$ is the transverse momentum of the test particle in the reaction plane. In this note, we shall refer to "flow" in the sense of the above equation.

We begin by examining the way the local particle density $\rho$ is calculated for the mean field dynamics in a Vlasov simulation. While the mean field is momentum dependent [12]- 14], we shall consider here, for illustrative purposes, a Skyrme-like parametrization that is a function of the density alone:

$$
U(\rho)=A \frac{\rho}{\rho_{0}}+B\left(\frac{\rho}{\rho_{0}}\right)^{\sigma},
$$

and take values of the parameters $A=-124 \mathrm{MeV}, B=70 \mathrm{MeV}$, and $\sigma=2$. This choice reproduces known nuclear matter properties, with a rather stiff compression modulus at saturation of $K_{0}=380 \mathrm{MeV}$.

We choose to represent the nucleon phase space distribution function $f(\vec{r}, \vec{p}, t)$ by an ensemble of test particles. If $f(\vec{r}, \vec{p}, t)$ is to satisfy the Vlasov equation, the equations of motion of a test particle with coordinates $\left(\vec{r}_{i}, \vec{p}_{i}\right)$ are given by Hamilton's equations of motion with potential (2):

$$
\dot{\vec{p}}_{i}=-\vec{\nabla}_{r} U\left(\rho\left(\vec{r}_{i}\right)\right) \quad \text { and } \quad \dot{\vec{r}}_{i}=\frac{\vec{p}_{i}}{\sqrt{m_{N}^{2}+p_{i}^{2}}}
$$

where $m_{N}$ is the free nucleon mass. The local particle density $\rho$ is often calculated on a grid, and the gradient obtained as a finite difference. In this procedure, each test particle counts a certain fraction towards the density of the cell it occupies, while neighboring cells receive a smaller contribution [15]. This stabilizes the numerics, but also introduces a nuclear surface whose thickness is roughly given by the grid size $\Delta x$. This grid size must be larger than the maximum distance traversed by a test particle in one time step, but small enough to be able to compute the gradients in Eq. (3) to sufficient accuracy.

Instead of "smearing" a test particle in steps over only the nearest neighboring cells, one may choose to supply each test particle with, say, a Gaussian density profile of constant width. This also introduces a finite surface thickness, but one 
that is well-defined and independent of the grid size. Thus one may study the effect of varying the nuclear surface thickness without the external numerical constraints imposed on the choice of $\Delta x$.

As an illustrative example, we consider flow in pure Vlasov dynamics as a function of the size of the grid for ${ }^{139} \mathrm{La}$ on ${ }^{139} \mathrm{La}$ collisions at a beam energy of $800 \mathrm{MeV} /$ nucleon and impact parameter $b=2.7 \mathrm{fm}$. Fig. 1 shows that $\left\langle w P_{x}\right\rangle$ depends very strongly on the grid size, i.e., the nuclear surface (dotted line). The extrapolated value to zero grid size (zero surface thickness) is twice as large as the value at $\Delta x=1 \mathrm{fm}$, a frequently used grid size. This strong dependence persists at beam energies of $200 \mathrm{MeV} /$ nucleon. At this energy, calculations with finite $\Delta x>0.5 \mathrm{fm}$ gave an overall attraction, whereas the extrapolation to $\Delta x=0$ predicted positive flow.

In Fig. 1, we also show the flow obtained with Gaussian test particle density profiles. The solid line represents the variation with $\Delta x$ for a surface thickness of $2 \mathrm{fm}$, while the dashed line is for a surface of $1 \mathrm{fm}$. Clearly, as long as the grid size is smaller than the Gaussian smearing width (i.e. the nuclear surface), the flow is independent of $\Delta x$. Also, the dotted line crosses the solid and dashed lines at $\Delta x \sim 2$ and $1 \mathrm{fm}$, respectively. This indicates that at least most of its rise with decreasing grid size is directly attributable to the changing surface thickness, and is not a "numerical artifact." We conclude that $\left\langle w P_{x}\right\rangle$ depends rather strongly on the surface thickness, a thinner surface producing more than a thicker one. Of course, quantitatively the results obtained here are not reliable, since we have, for instance, ignored the momentum dependence and hard collisions, but they do show the important influence of the nuclear surface.

The surface dependence of flow can be understood in terms of a simple potential scattering model [14]. Assuming that the nuclei pass through each other without changing their shape in phase space, the centers of mass of the nuclei move according to the Hamilton function

$$
H=\frac{P_{1}^{2}}{2 M}+\frac{P_{2}^{2}}{2 M}+\tilde{V}(R)
$$


where the potential $\tilde{V}$ is given by

$$
\tilde{V}(R)=\int V\left(\rho_{R}(r)\right) d r^{3}
$$

$M$ is the nuclear mass, $P_{1}$ and $P_{2}$ the center-of-mass momenta, and $R$ the separation of the nuclei. In (5), $V\left(\rho_{R}\right)$ is the potential energy density corresponding to Eq. (2)

$$
V(\rho)=\frac{A}{2} \frac{\rho^{2}}{\rho_{0}}+\frac{B}{\sigma+1} \frac{\rho^{\sigma+1}}{\rho_{0}^{\sigma}} .
$$

To describe nuclei with a surface, we choose the density profile to be

$$
\frac{\rho_{R}(r)}{\rho_{0}}=\left[1+\exp \left(|\vec{r}-\vec{R} / 2|-R_{0}\right) / a\right]^{-1}+\left[1+\exp \left(|\vec{r}+\vec{R} / 2|-R_{0}\right) / a\right]^{-1}
$$

where $R_{0}$ is the nuclear radius, and $4 a$ the surface thickness.

The potential (6) is shown in Fig. 2 for various values of the parameter $a$. As expected, for an increasing surface thickness, the potential $\tilde{V}$ decreases, and we expect a larger surface to produce less flow. This can be shown explicitly in the time evolution of the $\left\langle w P_{x}\right\rangle$ (see Fig. 3). We find reasonable agreement of the potential scattering model with the test particle Vlasov calculation at both values of the surface thickness. Of course, differences are seen in the details, and are expected because of the crude assumptions made in Eq. (4). For example, a Vlasov calculation shows that $E_{B a l}$ occurs between $200 \mathrm{MeV}$ and $300 \mathrm{MeV}$, depending on the surface, while the scattering model shows no zeros in the flow excitation function.

We now focus on the disappearance of flow in a more realistic calculation, i.e., a full BUU simulation that includes collisions. For the reaction ${ }^{139} \mathrm{La}$ on ${ }^{139} \mathrm{La}$ [1] we find that the balance energy is shifted by $\approx 10 \mathrm{MeV}$ when the surface thickness increases from $1 \mathrm{fm}$ to $2 \mathrm{fm}$. This is comparable to the shift expected when one changes from a stiff to a soft equation of state. For example, in ${ }^{40} \mathrm{Ar}$ on $\mathrm{V}$ reactions, $E_{B a l}$ changes by only $8 \mathrm{MeV}$ if the incompressibility is increased from $K_{0}=200 \mathrm{MeV}$ to $380 \mathrm{MeV}$ by adjusting the parameters in Eq. (2) [3].

This becomes even more apparent in smaller systems such as ${ }^{12} \mathrm{C}+{ }^{12} \mathrm{C}$, for which values of $E_{B a l}$ were recently measured at the National Superconducting Cyclotron Laboratory 16. Fig. 4 shows the flow obtained in simulations as a function of beam 
energy, for surface thicknesses of $1 \mathrm{fm}$ (left panel) and $2 \mathrm{fm}$ (right panel). The stiff EOS gives $E_{B a l} \approx 140 \mathrm{MeV}$ and $190 \mathrm{MeV}$, respectively, while the soft EOS yields $E_{B a l} \approx 170 \mathrm{MeV}$ and $220 \mathrm{MeV}$, respectively. [18]. The relative importance of the surface thickness is enhanced in smaller systems. Of course, the use of momentum dependent mean fields, a local Thomas-Fermi momentum space initialization, and values of the in-medium NN cross section will also influence the balance energy. For instance, it has been shown [3] that BUU calculations with reduced NN cross sections result in higher values of the balance energy.

Fig. 5 shows the balance energy as a function of the combined mass of the system. Experimental data (squares and diamond) suggests that the dependence of the balance energy on the combined mass, $A$, of the colliding nuclei follows a power law,

$$
E_{B a l}=x A^{-y}
$$

where the exponent $y$ has a numerical value of $0.33 \pm 0.04$. This power law dependence is reproduced by the BUU simulation (triangles and circles correspond to a soft and stiff EOS respectively) [19]. In this calculation, we have parametrized the the nucleon-nucleon cross sections in terms of a least squares fit to the experimental data of Ref. [17]. The resulting values are somewhat different from the iso-spin averaged expressions used at higher beam energies. In particular, $\sigma_{p p} \neq \sigma_{p n}$, and the cross sections are larger than the ones described in Ref. [15]. This results in a shift of the balance energy to lower values.

In Fig. 6, we investigate the dependence of the balance energy on the value of the in-medium nucleon-nucleon cross section, where we look for medium corrections beyond the effect of the Pauli principle on the outgoing scattering states. In previous studies the free nucleon-nucleon cross section was multiplied with an overall constant scaling factor [3, 7, 20]. However, this approach fails when one has collisions in lowdensity nuclear matter, where the in-medium cross section should approach its freespace value. A more realistic approach uses a Taylor expansion of the in-medium 
cross section in the density variable:

$$
\begin{aligned}
\sigma_{\mathrm{NN}}(\sqrt{s}, \rho) & =\sigma_{\mathrm{NN}}(\sqrt{s}, 0)+\left.\rho \frac{\partial \sigma_{N N}(\sqrt{s}, \rho)}{\partial \rho}\right|_{\rho=0}+\ldots \\
& =\left(1+\alpha_{1} \frac{\rho}{\rho_{0}}\right) \sigma_{\mathrm{NN}}(\sqrt{s}, 0)+\ldots
\end{aligned}
$$

where we have introduced the dimensionless parameter $\alpha_{1}$, given by:

$$
\alpha_{1}=\left.\rho_{0} \frac{\partial}{\partial \rho}\left\{\ln \sigma_{\mathrm{NN}}(\sqrt{s}, \rho)\right\}\right|_{\rho=0} .
$$

In principle, $\alpha_{1}$ is dependent on $\sqrt{s}$, but we have here - as a first approximation - taken $\alpha_{1}$ as an energy-independent constant. Fig. 6 shows the mass dependence of the calculated balance energy as a function of different values of $\alpha_{1}$, where we have used a soft nuclear equation of state. It is clear that we obtain the best overall agreement with the experimental data for a value of $\alpha_{1}=-0.2$, correponding to a $20 \%$ reduction of the nucleon-nucleon cross section at $\rho=\rho_{0}$. However, the powerlaw exponent $y$ from Eq. (8) depends on the choice of $\alpha_{1}$, with $\alpha_{1}=0$ (no medium modification) yielding the best agreement $(y=0.38 \pm 0.05)$ with the experimental value of $y=0.32$. For $\alpha_{1}=-0.1$ one extracts $y=0.42 \pm 0.03$, and for $\alpha_{1}=-0.2$ we obtain $y=0.47 \pm 0.03$. That $y$ increases with $\left|\alpha_{1}\right|$ is due to the fact that at higher beam energies higher densities are reached, and therfore the reduction of the in-medium cross section is stronger for the lighter systems, which have higher balance energies. This effect may, however, be at least partially compensated once one incorporates a more realistic energy dependence of $\alpha_{1}$.

In addition to effects mentioned previously, the proportionality constant $x$ (and, in principle, also the exponent y) in Eq. (8) also depends on the impact parameter $b$ [21], as can been seen in Fig. 7. For peripheral collisions, the contribution of the nucleon-nucleon collisions becomes relatively less important for the flow production than the mean field, because the repulsion generated by the nucleon-nucleon collisions is proportional to the overlap volume. We note that the calculations in Figs. 5 and 6 use only one impact parameter, obtained from the average value in the experiment, and proportionally scaled $b$ for calculations at mass numbers $A$ for 
which no data exists. Of course, for a quantitative comparison with experiment a impact parameter weighted averaged flow has to be considered, but calculating $E_{B a l}$ for several $A$ and $b$ is computationally prohibitive.

In conclusion, we have shown that in Vlasov and BUU simulations reliable results for flow must take into account the finite thickness of the nuclear surface. This is best done by giving the test particles a Gaussian density profile, with a width that is larger than the grid size used to obtain the density gradients. The value of the nuclear surface thickness has a strong effect on the balance energy. Therefore, more quantitative BUU predictions have to not only take into account the equation of state and medium effects on the nucleon-nucleon cross section, but also proper initial conditions in phase space and impact parameter averaging. In addition, we find that a realistic variation of the in-medium nucleon-nucleon cross-section with density has a clear effect on the mass dependence of the balance energy. We find best overall agreement with the experimental data for $\alpha_{1}=-0.2$, where $\alpha_{1}$ is defined in Eq. (10).

\section{Acknowledgements}

We are grateful to G.F. Bertsch and B.-A. Li for fruitful discussions. This work was supported the National Science Foundation under Grant No. 90-17077. W.B. acknowledges support from an NSF Presidential Faculty Fellow award, and D.K. was partially supported by the Studienstiftung des Deutschen Volkes.

*Present address: Institut für theoretische Physik II, Staudtstraße 7, W-85200 Erlangen, Germany. 


\section{References}

[1] D. Krofcheck et al., Phys. Rev. Lett. 63 (1989) 2028.

[2] G.D. Westfall et al., Nucl. Phys. A 519 (1990) 141c.

[3] C.A. Ogilvie et al. Phys. Rev. C 42 (1990) R10.

[4] J.P. Sulivan et al., Phys. Lett. B 249 (1990) 8.

[5] G.D. Westfall et al., submitted to Physical Review Letters (1992).

[6] J. Molitoris, D. Hahn, and H. Stöcker, Nucl. Phys. A 447 (1986) 13c.

[7] G.F. Bertsch, W.G. Lynch, and M.B. Tsang, Phys. Lett. B 189 (1987) 384.

[8] A. Bonasera and L.P. Csernai, Phys. Rev. Lett. 59 (1987) 630.

[9] P. Danielewicz and M. Gyulassy, Phys. Lett. B 129 (1983) 283.

[10] P. Danielewicz and G. Odyniec, Phys. Lett. B 157 (1985) 146.

[11] W.K. Wilson, R. Lacey, C.A. Ogilvie, and G.D. Westfall, Phys. Rev. C 45 (1992) 738 .

[12] C. Gale, G.F. Bertsch, and S. Das Gupta, Phys. Rev C 37 (1987) 1666.

[13] G.M. Welke et al., Phys. Rev. C 38 (1988) 2101.

[14] C. Gale, G.M. Welke, M. Prakash, S.J. Lee, and S. Das Gupta, Phys. Rev. C $41(1990) 1545$.

[15] G.F. Bertsch and S. Das Gupta, Phys. Rep. 160 (1988) 189.

[16] G.D. Westfall, "The disappearance of flow" Proceedings of the 8th Winter Workshop on Nuclear Dynamics, p. 196, 18-25 January, 1992, eds. W. Bauer and B. Back (World Scientific, 1992).

[17] Particle Data Group, Phys. Lett. B 204 (1988) 1. 
[18] Similar results for the balance energy are found by R.K. Tripathi, "Is there universality in flow phenomena in heavy ion collisions?" Proceedings of the 8th Winter Workshop on Nuclear Dynamics, p. 205, 18-25 January, 1992, eds. W. Bauer and B. Back (World Scientific, 1992);

R.K. Tripathy, L.W. Townsend, and F. Khan, Phys. Rev. C 47, R935 (1993).

[19] Tripathi et al. 18 have observed a similar scaling law using a microscopic optical model formalism. De la Mota et al. [20] also obtain this dependence, using the Landau-Vlasov method with a mean field derived from the Gogny force.

[20] V. de la Mota, F. Sebille, M. Farine, B. Remaud, and P. Schuck, Phys. Rev. C 46, 677 (1992).

[21] The impact parameter dependence of several observables has been systematically investigated by M.B. Tsang, G.F. Bertsch, W.G. Lynch, and M. Tohyama Phys. Rev. C 40 (1989) 1685. 


\section{Figure Captions}

Fig.1 Flow (as defined in Eq. (1)) versus grid size for pure Vlasov dynamics with smearing over neighboring cells only (dotted line). The dashed and solid lines show results if each test particle is given a Gaussian density profile of constant width. The dashed line corresponds to a nuclear surface thickness of $1 \mathrm{fm}$, the solid line to $2 \mathrm{fm}$. All curves shown are for ${ }^{139} \mathrm{La}+\mathrm{La}, E=800 \mathrm{MeV} /$ nucleon, and $b=2.7 \mathrm{fm}$.

Fig.2 Potential Eq. (5) as a function of the separation of the two nuclei. The radius $R_{0}=5.8 \mathrm{fm}$ corresponds to ${ }^{139} \mathrm{La}$.

Fig.3 The time evolution of flow for pure Vlasov dynamics (solid line), and the potential scattering model, Eq. (四) (dashes). The surface thicknesses are $1 \mathrm{fm}$ (upper two curves) and $2 \mathrm{fm}$ (lower two curves), and the system is as for Fig. 1.

Fig.4 Flow versus beam energy in the vicinity of the zeros of the excitation function. The system is ${ }^{12} \mathrm{C}+{ }^{12} \mathrm{C}$ at an impact parameter $b=1.4 \mathrm{fm}$. The left panel is for a surface thickness of $1 \mathrm{fm}$, the right for $2 \mathrm{fm}$. Both panels show results for stiff (diamonds) and soft equations of state (plus signs).

Fig.5 The calculated values of $E_{B a l}$ as a function of the mass of the system. Only symmetric systems are considered. Diamonds and circles correspond to a soft and stiff EOS, respectively. For comparison, experimental data from Ref. [5] (squares) [5] are shown. The solid and dashed curves are power-law fits to the calculations and data.

Fig.6 Same as Fig. 5, but varying the in-medium cross section according to Eq. (9) with $\alpha_{1}$ defined in Eq. (10). For all calculations, a soft equation of state was used. The lines represent power-law fits to the calculations and data.

Fig.7 The balance energy for $\mathrm{Cl}+\mathrm{Cl}$ as a function of impact parameter. 